Review

\title{
The Four-Parameters Wright Function of the Second kind and its Applications in FC
}

\author{
Yuri Luchko
}

Department of Mathematics, Physics, and Chemistry, Beuth Technical University of Applied Sciences Berlin, Luxemburger Str. 10, 13353 Berlin, Germany; luchko@beuth-hochschule.de

Received: 20 May 2020; Accepted: 10 June 2020; Published: 12 June 2020

check for updates

\begin{abstract}
In this survey paper, we present both some basic properties of the four-parameters Wright function and its applications in Fractional Calculus. For applications in Fractional Calculus, the four-parameters Wright function of the second kind is especially important. In the paper, three case studies illustrating a wide spectrum of its applications are presented. The first case study deals with the scale-invariant solutions to a one-dimensional time-fractional diffusion-wave equation that can be represented in terms of the Wright function of the second kind and the four-parameters Wright function of the second kind. In the second case study, we consider a subordination formula for the solutions to a multi-dimensional space-time-fractional diffusion equation with different orders of the fractional derivatives. The kernel of the subordination integral is a special case of the four-parameters Wright function of the second kind. Finally, in the third case study, we shortly present an application of an operational calculus for a composed Erdélyi-Kober fractional operator for solving some initial-value problems for the fractional differential equations with the left- and right-hand sided Erdélyi-Kober fractional derivatives. In particular, we present an example with an explicit solution in terms of the four-parameters Wright function of the second kind.
\end{abstract}

Keywords: four-parameters Wright function of the second kind; one-dimensional time-fractional diffusion-wave equation; scale-invariant solutions; multi-dimensional space-time-fractional diffusion equation; subordination formula; left- and right-hand sided Erdélyi-Kober fractional derivatives

MSC: 26A33; 33E20; 30C15; 30D15; 45J05; 45K05; 44A20

\section{Introduction}

In calculus, differential equations, and mathematical physics both elementary and most of the special functions can be expressed in terms of the so-called generalized hypergeometric function ${ }_{p} F_{q}$ that is defined as the following series (in the case it converges):

$$
{ }_{p} F_{q}\left(a_{1}, \ldots, a_{p} ; b_{1}, \ldots, b_{q} ; z\right):=\sum_{k=0}^{\infty} \frac{\prod_{n=1}^{p}\left(a_{n}\right)_{k}}{\prod_{n=1}^{q}\left(b_{n}\right)_{k}} \frac{z^{k}}{k !}
$$

with the Pochhammer symbol $(z)_{k}, k \in \mathbb{N}$ given by the formula

$$
(z)_{k}=\frac{\Gamma(z+k)}{\Gamma(k)}=\prod_{n=0}^{k-1}(z+n) .
$$

In particular, all elementary functions can be represented in terms of the famous hypergeometric Gauss function ${ }_{2} F_{1}$. Other particular cases and properties of the generalized hypergeometric function can be found in [1]. 
If $p \leq q$, the series at the right-hand side of the formula (1) is absolutely convergent for all values of $z \in \mathbb{C}$. For $p=q+1$, the series converges for $|z|<1$ and for $|z|=1$ under some additional conditions. If $p>q+1$, the series is divergent.

To overcome this restriction and to somehow define the function ${ }_{p} F_{q}$ in the case $p>q+1$, in [2] Meijer introduced a very general special function presently known in the literature as the $G$-function. For definition, properties, and particular cases of the $G$-function we refer the readers to [1].

However, it turned out that the special functions of Fractional Calculus (FC) belong in general neither to particular cases of the generalized hypergeometric function ${ }_{p} F_{q}$ nor to particular cases of the Meijer $G$-function. They are particular cases of the more general generalized Wright or Fox-Wright functions or the Fox $H$-function ([1,3-7]).

The probably most used and important special functions of FC are the Mittag-Leffler function and its generalizations and the Wright function and its generalizations. For the theory of the Mitag-Leffler type functions and their applications we refer the readers to the book [3] and the recent survey [8] (see also numerous references therein). As to the Wright function and its generalizations, parts of their theory and some applications were presented in [5,6,9-29].

In this paper, the focus is on the four-parameters Wright function and its applications in FC. Depending on the signs of the parameters, we distinguish between the four-parameters Wright function of the first kind and of the second kind. The four-parameters Wright function of the first kind was first considered by Fox in [30] and by Wright in [28] (more precisely, this function was a particular case of the generalized Fox-Wright function that satisfies some conditions). In [12], the four-parameters Wright function of the first kind was employed as a kernel of an integral transform. It is the first application of this function known to the author. Another useful application of the four-parameters Wright function of the first kind was presented in [31], where the authors developed an operational calculus for an integral operator with the Gauss hypergeometric function as the kernel. This operational calculus was then used for derivation of the exact solutions of some integral equations of Volterra-type with the Gauss hypergeometric function in the kernel in terms of the four-parameters Wright function of the first kind.

As to the four-parameters Wright function of the second kind, it was first introduced in Luchko and Gorenflo [18]. Luchko and Gorenflo also provided some important properties of this function including its integral representation via the Mittag-Leffler function and its asymptotic behavior. Moreover, they applied the four-parameters Wright function of the second kind for derivation of the explicit analytical scale-invariant solutions to a one-dimensional space-time fractional diffusion equation. In this paper, some important results from [18] and the subsequent publications $[5,15,32,33]$ will be revisited.

The rest of the paper is organized as follows: In the 2nd Section, we introduce the Wright function, the four-parameters Wright function, and the generalized Wright or Fox-Wright function and provide some of their important properties with the special focus on the four-parameters Wright function of the second kind. In the 3rd Section, three examples of applications of the four-parameters Wright function of the second kind in FC are presented. The first example deals with analysis of the scale-invariant solutions to a one-dimensional time-fractional diffusion-wave equation $([14,15])$. It turns out that they can be represented in terms of the Wright function of the second kind and the four-parameters Wright function of the second kind. The second example is devoted to a subordination formula for the solutions to a multi-dimensional space-time-fractional diffusion equation with different orders of the fractional derivatives ([33]). The kernel of the subordination integral is a special case of the four-parameters Wright function of the second kind that is non-negative and can be interpreted as a probability density function. In the third example, we present an application of the operational method suggested in [32] for derivation of solution to an initial-value problem for a fractional differential equation with the left- and right-hand sided Erdélyi-Kober fractional derivatives in terms of the four-parameters Wright function of the second kind. 


\section{The Four-Parameters Wright Function}

The generalized hypergeometric function ${ }_{p} \Psi_{q}$ presently known as the generalized Wright or Fox-Wright function was introduced and investigated by Fox in [30] and by Wright in [28]. It is defined by the convergent series

$$
{ }_{p} \Psi_{q}\left[\begin{array}{c}
\left(a_{1}, A_{1}\right), \ldots,\left(a_{p}, A_{p}\right) \\
\left(b_{1}, B_{1}\right) \ldots\left(b_{q}, B_{q}\right)
\end{array} ; z\right]:=\sum_{k=0}^{\infty} \frac{\prod_{i=1}^{p} \Gamma\left(a_{i}+A_{i} k\right)}{\prod_{i=1}^{q} \Gamma\left(b_{i}+B_{i} k\right)} \frac{z^{k}}{k !}, z \in \mathbb{C}
$$

with $a_{i} \in \mathbb{R}, A_{i}>0, i=1, \ldots, p, b_{i} \in \mathbb{R}, B_{i}>0, i=1, \ldots, q$. In the case $A_{i}=1, i=1, \ldots, p$, $B_{i}=1, i=1, \ldots, q$, the generalized Wright function coincides with the generalized hypergeometric function (1) up to a constant factor. Even more, in the case of the positive rational parameters $A_{i} \in \mathbb{Q}$, $i=1, \ldots, p, B_{i} \in \mathbb{Q}, i=1, \ldots, q$, the generalized Wright function can be represented as a final sum of the generalized hypergeometric functions with the power functions weights. Say, in the case $p=0$, $q=1$, and $B_{1}=\frac{n}{m} \in \mathbb{Q}, n, m>0$, we have the following representation ([14]):

$$
{ }_{0} \Psi_{1}\left[\begin{array}{c}
- \\
\left(\beta, \frac{n}{m}\right)
\end{array} ; z\right]=\sum_{p=0}^{m-1} \frac{z^{p}}{p ! \Gamma\left(\beta+\frac{n}{m} p\right)}{ }_{0} F_{n+m-1}\left(-; \Delta\left(n, \frac{\beta}{n}+\frac{p}{m}\right), \Delta^{*}\left(m, \frac{p+1}{m}\right) ; \frac{z^{m}}{m^{m} n^{n}}\right)
$$

where $\Delta(k, a)$ and $\Delta^{*}(k, a)$ are defined by

$$
\Delta(k, a)=\left\{a, a+\frac{1}{k}, \ldots, a+\frac{k-1}{k}\right\}, \Delta^{*}(k, a)=\Delta(k, a) \backslash\{1\} .
$$

In the case of the formula (3), the set $\Delta^{*}(k, a)$ is correctly defined since 1 is an element of any set $\Delta\left(m, \frac{p+1}{m}\right), 0 \leq p \leq m-1$. The method employed in [14] for derivation of the formula (3) can be also applied to obtain similar but of course even more complicated representations for the function ${ }_{p} \Psi_{q}$ with the positive rational parameters $A_{i} \in \mathbb{Q}, i=1, \ldots, p, B_{i} \in \mathbb{Q}, i=1, \ldots, q$ in terms of the generalized hypergeometric function (1).

It is worth mentioning that both in [28,30], the parameters $A_{i}$ and $B_{i}$ were supposed to be positive real numbers. However, in [29], Wright considered a particular case of the function ${ }_{p} \Psi_{q}$ with $p=0$ and $q=1$ and the coefficient $B_{1}$ being any real number greater than -1 . Presently this function is called the Wright function. Following Wright, it is denoted by $\phi(\rho, \beta ; z)$ :

$$
\phi(\rho, \beta ; z):={ }_{0} \Psi_{1}\left[\begin{array}{c}
- \\
(\beta, \rho)
\end{array} ; z=\sum_{k=0}^{\infty} \frac{z^{k}}{k ! \Gamma(\beta+\rho k)}, z \in \mathbb{C}, \rho>-1, \beta \in \mathbb{C} .\right.
$$

For $\rho>-1$, the series at the right-hand side of the formula (4) is convergent for all $z \in \mathbb{C}$. It is also convergent for $\rho=-1$ and $|z|<1$ and for $\rho=-1$ and $|z|=1$ under the condition $\Re(\beta)>1$. However, the Wright function is an entire function only in the case $\rho>-1$ and thus this condition is usually included into its definition.

In $[3,19]$, the function (4) with the positive parameter $\rho$ was called the Wright function of the first kind, whereas in the case of the negative parameter $\rho(0>\rho>-1)$ it was called the Wright function of the second kind. In the case $\rho=0$, the Wright function is reduced to the exponential function:

$$
\phi(0, \beta ; z)=\sum_{k=0}^{\infty} \frac{z^{k}}{k ! \Gamma(\beta)}=\frac{e^{z}}{\Gamma(\beta)}
$$

In analogy to the Wright function (4), the generalized Wright function (2) can be considered also in the case, some or even all of the parameters $A_{i}$ and $B_{i}$ are negative numbers. The well-known asymptotic behavior of the Euler Gamma-function allows determination of the convergence radius of the series at the right-hand side of (2): it is absolutely convergent for all $z \in \mathbb{C}$ under the condition 
$\Delta>-1$, where $\Delta$ is determined by the parameters of the generalized Wright function as follows (see, e.g., $[3,16,34]$ ):

$$
\Delta=\sum_{i=1}^{q} B_{i}-\sum_{i=1}^{p} A_{i}, \delta=\prod_{i=1}^{p}\left|A_{i}\right|^{-A_{i}} \prod_{i=1}^{q}\left|B_{i}\right|^{B_{i}}, \mu=\sum_{i=1}^{q} b_{i}-\sum_{i=1}^{p} a_{i}+\frac{p-q}{2} .
$$

In the case $\Delta>-1$, the function (2) is an entire function. However, in the case $\Delta=-1$, the series at the right-hand side of (2) is also absolutely convergent for $|z|<\delta$ and for $|z|=\delta$ under the condition $\Re(\mu)>1 / 2$ (see [34] for details).

In this paper, we mainly deal with another important particular case of the generalized Wright function (2), specifically with the so-called four-parameters Wright function:

$$
W_{\left(\rho_{1}, \beta_{1}\right),\left(\rho_{2}, \beta_{2}\right)}(z):={ }_{1} \Psi_{2}\left[\begin{array}{c}
(1,1) \\
\left(\beta_{1}, \rho_{1}\right)\left(\beta_{2}, \rho_{2}\right)
\end{array} ; z\right] .
$$

According to the definition of the generalized Wright function, the series representation of the four-parameters Wright function is as follows:

$$
W_{\left(\rho_{1}, \beta_{1}\right),\left(\rho_{2}, \beta_{2}\right)}(z)=\sum_{k=0}^{\infty} \frac{z^{k}}{\Gamma\left(\beta_{1}+\rho_{1} k\right) \Gamma\left(\beta_{2}+\rho_{2} k\right)}, \rho_{1}, \rho_{2} \in \mathbb{R}, \beta_{1}, \beta_{2} \in \mathbb{C}, z \in \mathbb{C} .
$$

For $\rho_{1}+\rho_{2}>0$, the series at the right-hand side of (8) is absolutely convergent $\forall z \in \mathbb{C}$. For $\rho_{1}+$ $\rho_{2}=0$, the series is absolutely convergent for $|z|<1$ and for $|z|=1$ under the condition $\Re\left(\beta_{1}+\beta_{2}\right)>$ 2. Finally, the series is divergent for any $z \neq 0$ in the case $\rho_{1}+\rho_{2}<0$.

Without any loss of generality, in what follows we always suppose that the condition $\rho_{1} \geq \rho_{2}$ holds true in the definition of the four-parameters Wright function. This assumption will lead to simpler formulations of some results concerning the four-parameters Wright function. Moreover, we will distinguish between the four-parameters Wright function of the first kind $\left(\rho_{2}>0\right)$ and of the second kind $\left(\rho_{2}<0\right)$. The properties and applications of the four-parameters Wright function of the second kind are very different from those of the function of the first kind. Thus, we found it appropriate to introduce a separate notation for the four-parameters Wright function of the second kind:

$$
\Phi_{\left(\rho_{1}, \beta_{1}\right),\left(\rho_{2}, \beta_{2}\right)}(z):=W_{\left(\rho_{1}, \beta_{1}\right),\left(\rho_{2}, \beta_{2}\right)}(z), \rho_{2}<0 .
$$

The notation $W_{\left(\rho_{1}, \beta_{1}\right),\left(\rho_{2}, \beta_{2}\right)}$ is kept for the four-parameters Wright function (including the cases of the functions of the first and of the second kinds).

In what follows, we always suppose that the condition $\rho_{1}+\rho_{2}>0$ is satisfied. This condition along with the inequality $\rho_{1} \geq \rho_{2}$ leads to the inequality $\rho_{1}>0$. Thus, the parameter $\rho_{1}$ of the four-parameters Wright function is always positive, whereas the parameter $\rho_{2}$ is positive in the case of the function of the first kind and negative in the case of the function of the second kind. In the case $\rho_{2}=0$, the four-parameters Wright function is reduced to the two-parameters Mittag-Leffler function:

$$
W_{\left(\rho_{1}, \beta_{1}\right),\left(0, \beta_{2}\right)}(z)=\frac{1}{\Gamma\left(\beta_{2}\right)} E_{\rho_{1}, \beta_{1}}(z)=\frac{1}{\Gamma\left(\beta_{2}\right)} \sum_{k=0}^{\infty} \frac{z^{k}}{\Gamma\left(\beta_{1}+\rho_{1} k\right)} .
$$

For the theory and applications of the two-parameters Mittag-Leffler function we refer to the book [3]; in this paper we do not consider this function. Please note that in [3] the function (7) is called the generalized Mittag-Leffler function or the four-parametric Mittag-Leffler function.

Another important particular case of the four-parameters Wright function (7) is the Wright function (4):

$$
W_{(1,1),(\rho, \beta)}(z)=\phi(\rho, \beta ; z) \text {. }
$$


For the properties and applications of the Wright function we refer to the recent survey [5], see also the references therein.

As already mentioned, the four-parameters Wright function is an entire function provided the condition $\rho_{1}+\rho_{1}>0$ holds true.

Theorem 1. Let the condition $\rho_{1}+\rho_{1}>0$ be satisfied. Then the four-parameters Wright function is an entire function of the variable $z$. Its order $p$ and type $\sigma$ are given by the relations

$$
p=\frac{1}{\rho_{1}+\rho_{2}}, \sigma=\frac{\rho_{1}+\rho_{2}}{\left(\rho_{1}^{\rho_{1}}\left|\rho_{2}\right|^{\rho_{2}}\right)^{\frac{1}{\rho_{1}+\rho_{2}}}} .
$$

The proof of the theorem is based on the Stirling formula for the asymptotic of the Gamma-function and can be found in $[3,12,16]$.

Since it is a function of the hypergeometric type, the four-parameters Wright function possesses a very useful Mellin-Barnes integral representation ([35]):

$$
W_{\left(\rho_{1}, \beta_{1}\right),\left(\rho_{2}, \beta_{2}\right)}(z)=\frac{1}{2 \pi i} \int_{L_{-\infty}} \frac{\Gamma(s) \Gamma(1-s)}{\Gamma\left(\beta_{1}-\rho_{1} s\right) \Gamma\left(\beta_{2}-\rho_{2} s\right)}(-z)^{-s} d s,
$$

where $L_{-\infty}$ is a left loop located in a horizontal strip. It goes from the point $-\infty+i y_{1}$ to the point $-\infty+i y_{2}$ with $y_{1}<0<y_{2}$ and separates the poles of the Gamma-function $\Gamma(s)$ (the points $s_{k}=$ $0,-1,-2, \ldots)$ from the poles of the Gamma-function $\Gamma(1-s)$ (the points $\left.s_{l}=1,2,3, \ldots\right)$.

The formula (13) can be easily proved by evaluating the Mellin-Barnes integral taking into account the Jordan lemma, the formula

$$
\operatorname{res}_{s=-k} \Gamma(s)=\frac{(-1)^{k}}{k !}, k=0,1,2, \ldots,
$$

the known asymptotic of the Gamma-function, and the Cauchy residue theorem.

Depending on the sign of the parameter $\rho_{2}$ (the parameter $\rho_{1}$ is always positive), the right-hand side of the representation (13) can be interpreted as the Fox $H$-function:

$$
\begin{gathered}
W_{\left(\rho_{1}, \beta_{1}\right),\left(\rho_{2}, \beta_{2}\right)}(z)=H_{1,3}^{1,1}\left(\begin{array}{c}
(0,1) \\
(0,1),\left(1-\beta_{1}, \rho_{1}\right),\left(1-\beta_{2}, \rho_{2}\right)
\end{array} \mid-z\right), \rho_{2}>0, \\
\Phi_{\left(\rho_{1}, \beta_{1}\right),\left(\rho_{2}, \beta_{2}\right)}(z)=H_{2,2}^{1,1}\left(\begin{array}{c}
(0,1),\left(\beta_{2},-\rho_{2}\right) \\
(0,1),\left(1-\beta_{1}, \rho_{1}\right)
\end{array} \mid-z\right), \rho_{2}<0 .
\end{gathered}
$$

It is worth mentioning that both the Mellin-Barnes integral representation (13) and the Fox $H$-function representations (15) and (16) can be used for derivation of several useful properties of the four-parameters Wright function including its particular cases for the rational values of the parameters $([1,3,5])$ or its asymptotic behavior $([6,7])$.

Because the focus of this paper is on applications of the four-parameters Wright function of the second kind in FC, in the rest of this section we mainly restrict ourselves to a short discussion of its important properties. For the proofs, we refer the interested readers to [18].

A very useful integral representation of the four-parameters Wright function is given in the following theorem:

Theorem 2 ([18]). The four-parameters Wright function possesses the following integral representation in terms of the two parameters Mittag-Leffler function (10):

$$
W_{\left(\rho_{1}, \beta_{1}\right),\left(\rho_{2}, \beta_{2}\right)}(z)=\frac{1}{2 \pi i} \int_{\gamma(\varepsilon ; \varphi)} e^{\zeta} \zeta^{-\beta_{2}} E_{\rho_{1}, \beta_{1}}\left(z \zeta^{-\rho_{2}}\right) d \zeta,
$$


where $\gamma(\varepsilon ; \varphi)\left(\epsilon>0, \frac{\pi}{2}<\varphi \leq \pi\right)$ is a contour in the complex plane with the nondecreasing arg $\zeta$ that consists of the ray $\arg \zeta=-\varphi,|\zeta| \geq \varepsilon$, the arc $-\varphi \leq \arg \zeta \leq \varphi$ of the circle $|\zeta|=\varepsilon$, and the ray $\arg \zeta=\varphi,|\zeta| \geq \varepsilon$.

In the case of the four-parameters Wright function of the second kind, the integration contour $\gamma(\varepsilon ; \varphi)$ in Theorem 2 can be replaced by a simpler one:

Theorem 3 ([18]). For any $k_{0} \in \mathbb{N}$ satisfying the condition $k_{0}>\max \left\{-1, \Re\left(\left(1-\beta_{2}\right) /\left(-\rho_{2}\right)\right)\right\}$, the four-parameters Wright function of the second kind can be represented as follows:

$$
\begin{aligned}
\Phi_{\left(\rho_{1}, \beta_{1}\right),\left(\rho_{2}, \beta_{2}\right)}(z)= & \sum_{k=0}^{k_{0}} \frac{z^{k}}{\Gamma\left(\beta_{1}+\rho_{1} k\right) \Gamma\left(\beta_{2}+\rho_{2} k\right)}+ \\
& \frac{1}{2 \pi i} \int_{L_{-}}\left(e^{\zeta} \zeta^{-\beta_{2}} E_{\rho_{1}, \beta_{1}}\left(z \zeta^{-\rho_{2}}\right)-\sum_{k=0}^{k_{0}} \frac{\left(z \zeta^{-\rho_{2}}\right)^{k}}{\Gamma\left(\beta_{1}+\rho_{1} k\right)}\right) d \zeta,
\end{aligned}
$$

where $L_{-}$is a cut in the complex $\zeta$-plane along the negative real semi-axis.

Remark 1. As already mentioned, for $\beta_{1}=\rho_{1}=1$, the four-parameters Wright function is reduced to the Wright function (4) and the integral representation (17) with $\rho_{2}=\rho>-1$ and $\beta_{2}=\beta \in \mathbb{R}$ takes the well-known form

$$
\phi(\rho, \beta ; z)=\frac{1}{2 \pi i} \int_{\gamma(\varepsilon ; \varphi)} \exp \left\{\zeta+z \zeta^{-\rho}\right\} \zeta^{-\beta} d \zeta .
$$

This integral representation was obtained by Wright in [27,29] and then used for derivation of the asymptotic behavior of the Wright function. In particular, he showed that the Wright function of the second kind has an algebraic asymptotic expansion on the positive real semi-axis provided the condition $1 / 3<-\rho<1$ holds true $(K=0,1,2, \ldots)$ :

$$
\phi(\rho, \beta ; x)=\sum_{k=0}^{K-1} \frac{x^{(\beta-1-k) /(-\rho)}}{(-\rho) \Gamma(k+1) \Gamma(1+(\beta-l-k) /(-\rho))}+O\left(x^{(\beta-1-K) /(-\rho)}\right), x \rightarrow+\infty .
$$

For the four-parameters Wright function of the second kind, a similar result was obtained in [18].

Theorem 4 ([18]). Under the condition $\rho_{1} / 3<-\rho_{2}<\rho_{1} \leq 2$, the four-parameters Wright function of the second kind has the following asymptotic on the positive real semi-axis:

$$
\begin{gathered}
\Phi_{\left(\rho_{1}, \beta_{1}\right),\left(\rho_{2}, \beta_{2}\right)}(x)=\sum_{k=0}^{K-1} \frac{x^{\left(\beta_{2}-1-k\right) /\left(-\rho_{2}\right)}}{\left(-\rho_{2}\right) \Gamma(k+1) \Gamma\left(\beta_{1}+\rho_{1}\left(\beta_{2}-1-k\right) /\left(-\rho_{2}\right)\right)}- \\
\sum_{p=1}^{P} \frac{x^{-p}}{\Gamma\left(\beta_{1}-\rho_{1} p\right) \Gamma\left(\beta_{2}-\rho_{2} p\right)}+O\left(x^{\left(\beta_{2}-1-K\right) /\left(-\rho_{2}\right)}\right)+O\left(x^{-1-P}\right), \quad x \rightarrow+\infty
\end{gathered}
$$

for any $K=0,1,2, \ldots$, and $P=0,1,2, \ldots$

For geometric properties of the four-parameters Wright function we refer the interested readers to the very recent paper [11].

\section{Applications of the Four-Parameters Wright Function of the Second Kind}

In this section, we consider three examples of applications of the four-parameters Wright function of the second kind in FC.

The first example concerns the well-studied one-dimensional time-fractional diffusion-wave equation with the Caputo derivative. For analytical treatment of this equation, the Wright functions of the second kind play a fundamental role $([10,14,15,19,36])$. Say, the fundamental solution to this 
equation can be expressed in terms of some special cases of the Wright function of the second kind (so-called Mainardi auxiliary functions). However, it turns out that the formulas for the scale-invariant solutions to the one-dimensional diffusion-wave equation involve both the Wright function of the second kind and the four-parameters Wright function of the second kind.

In the second example, we deal with a subordination formula for solutions to a multi-dimensional space-time-fractional diffusion equation ([33]). This equation is obtained from the diffusion equation by replacing the first order time derivative by the Caputo fractional derivative and the Laplace operator by the fractional Laplacian. This time, it is the four-parameters Wright function of the second kind that is of importance for this equation. In particular, a special case of the four-parameters Wright function of the second kind appears in the kernel of a subordination formula that connects the solution operators of this equation with different orders of the fractional derivatives to the classical solution of the conventional diffusion equation. Moreover, this kernel function is non-negative and can be interpreted as a probability density function.

The third example deals with the ordinary fractional differential equations that contain both the left- and the right-hand sided fractional derivatives. In [32], an operational method for the so-called composed Erdélyi-Kober fractional derivatives was suggested and applied for derivation of the analytical solutions to the initial-value problems for a special class of such equations. In this section, we present an equation of this sort with an explicit solution expressed in terms of the four-parameters Wright function of the second kind.

\subsection{Scale-Invariant Solutions to the One-Dimensional Time-Fractional Diffusion-Wave Equation}

In this subsection, we deal with the fractional diffusion-wave equation, which is obtained from the conventional diffusion or wave equation by replacing the first- or second-order time derivative, respectively, by the Caputo fractional derivative:

$$
\frac{\partial^{\alpha} u(x, t)}{\partial t^{\alpha}}=\frac{\partial^{2} u(x, t)}{\partial x^{2}}, 1<\alpha<2, t>0, x>0 .
$$

The Caputo fractional derivative of order $\alpha, 1<\alpha<2$, is defined as follows:

$$
\frac{\partial^{\alpha} u(x, t)}{\partial t^{\alpha}}=\frac{1}{\Gamma(2-\alpha)} \int_{0}^{t}(t-\tau)^{1-\alpha} \frac{\partial^{2} u(x, \tau)}{\partial \tau^{2}} d \tau .
$$

In particular, we are interested in the scale-invariant solutions to this equation. First, we introduce some basic notions concerning the similarity method for the general equation

$$
F(u)=0, u=u(x, t) .
$$

A one-parameter family of scaling transformations, denoted by $T_{\lambda}$, is called a transformation of the $(x, t, u)$-space of the form

$$
\bar{x}=\lambda^{a} x, \quad \bar{t}=\lambda^{b} t, \quad \bar{u}=\lambda^{c} u,
$$

where $a, b$, and $c$ are some constants and $\lambda$ is a real parameter restricted to an open interval $I$ containing the value $\lambda=1$.

The general Equation (24) is called invariant under the one-parameter family $T_{\lambda}$ of scaling transformations (25) if and only if $T_{\lambda}$ translates any solution $u$ of (24) to a solution $\bar{u}$ of the same equation:

$$
F(\bar{u})=0 \quad \text { if } \quad \bar{u}=T_{\lambda} u .
$$


A real-valued function $\eta(x, t, u)$ is called an invariant of the one-parameter family $T_{\lambda}$ of scaling transformations if it is unaffected by the transformations from $T_{\lambda}$ :

$$
\eta\left(T_{\lambda}(x, t, u)\right)=\eta(x, t, u) \quad \text { for all } \quad \lambda \in I .
$$

The general theory ([37]) says that on the half-space $\{(x, t, u): x>0, t>0\}$, the invariants of the scaling transformations (25) are provided by the functions

$$
\eta_{1}(x, t, u)=x t^{-a / b}, \eta_{2}(x, t, u)=t^{-c / b} u .
$$

Say, let the Equation (24) be a second-order partial differential equation

$$
G\left(x, t, u, u_{x}, u_{t}, u_{x x}, u_{t t}, u_{x t}\right)=0 .
$$

If this equation is invariant under the family $T_{\lambda}$ of scaling transformations (25), then the substitution

$$
u(x, t)=t^{c / b} v(z), \quad z=x t^{-a / b}
$$

reduces the Equation (28) to a second-order ordinary differential equation

$$
g\left(z, v, v^{\prime}, v^{\prime \prime}\right)=0 .
$$

In $[9,14,15,18]$, the scale-invariant solutions for the equation of type (22) with the fractional derivatives in the Caputo and Riemann-Liouville sense and for the more general time- and space-fractional partial differential equations were obtained. In all cases, these solutions were expressed in terms of the Wright function of the second kind and the four-parameters Wright function of the second kind. In what follows, we present some of these results for the Equation (22).

The group of scaling transformations for the fractional diffusion-wave Equation (22) can be determined in explicit form.

Theorem 5 ([9]). The group of scaling transformations of the Equation (22) has the form

$$
T_{\lambda} \circ(x, t, u)=\left(\lambda x, \lambda^{\frac{2}{\alpha}} t, \lambda^{c} u\right)
$$

with an arbitrary constant $c \in \mathbb{R}$ and its invariants are given by the formulas

$$
\eta_{1}(x, t)=x t^{-\alpha / 2}, \eta_{2}(x, t, u)=t^{-c \alpha / 2} u .
$$

In what follows, for the sake of convenience, we use the notation $\gamma=c \alpha / 2$.

The general theory of the Lie groups ([37]) and Theorem 5 ensure that the scale-invariant solutions of the Equation (22) have the form

$$
u(x, t)=t^{\gamma} v(y), y=x t^{-\alpha / 2}, \gamma=c \alpha / 2 .
$$

Substitution of the function $u$ from the formula (32) into the partial fractional differential Equation (22) transforms it into an ordinary fractional differential equation with an unknown function $v(y)$. More precisely, the following result holds true:

Theorem 6 ([9]). The scale-invariant solutions of the Equation (22) in the form (32) satisfy the equation

$$
\left({ }_{*} P_{2 / \alpha}^{\gamma-1, \alpha} v\right)(y)=v^{\prime \prime}(y), y>0,
$$


where the operator ${ }_{*} P_{2 / \alpha}^{\gamma-1, \alpha}$ is the Caputo type modification of the right-hand sided Erdélyi-Kober fractional derivative defined by

$$
\left({ }_{*} P_{\delta}^{\tau, \alpha} g\right)(y):=\left(K_{\delta}^{\tau, n-\alpha} \prod_{j=0}^{n-1}\left(\tau+j-\frac{1}{\delta} u \frac{d}{d u}\right) g\right)(y), y>0, \delta>0, n-1<\alpha \leq n \in \mathbb{N} .
$$

The operator $K_{\delta}^{\tau, \alpha}, \alpha>0$ is the right-hand sided Erdélyi-Kober fractional integral defined by

$$
\left(K_{\delta}^{\tau, \alpha} g\right)(y):=\frac{1}{\Gamma(\alpha)} \int_{1}^{\infty}(u-1)^{\alpha-1} u^{-(\tau+\alpha)} g\left(y u^{1 / \delta}\right) d u .
$$

For $\alpha=1$ and $\alpha=2$, the fractional diffusion-wave Equation (22) is reduced to the conventional one-dimensional diffusion or wave equation, respectively. The Equation (33) for the scale-invariant solutions of (22) is an ordinary differential equation, not a fractional one. In the case $\alpha=1$ (the diffusion equation) we have the representation

$$
\left(* P_{2}^{\gamma, 1} v\right)(y)=\left(\gamma-\frac{1}{2} y \frac{d}{d y}\right) v(y)
$$

and the Equation (33) takes the well-known form

$$
v^{\prime \prime}(z)+\frac{1}{2} y v^{\prime}(y)-\gamma v(y)=0 .
$$

In the case $\alpha=2$ (the wave equation) we get the formula

$$
\left({ }_{*} P_{1}^{\gamma-1,2} v\right)(y)=\left(\gamma-1-y \frac{d}{d y}\right)\left(\gamma-y \frac{d}{d y}\right) v(y)=y^{2} v^{\prime \prime}(y)-2(\gamma-1) y v^{\prime}(y)+\gamma(\gamma-1) v(y)
$$

and the equation (33) is transformed to the following ODE:

$$
\left(y^{2}-1\right) v^{\prime \prime}(y)-2(\gamma-1) y v^{\prime}(y)+\gamma(\gamma-1) v(y)=0 .
$$

These both cases are discussed in detail in [37].

It turns out that the Equation (34) can be solved in explicit form in terms of the Wright function of the second kind and the four-parameters Wright function of the second kind.

Theorem 7 ([14]). The scale-invariant solutions of the fractional diffusion-wave Equation (22) are given by the formulas

$$
u(x, t)=C_{1} t^{\gamma} \phi\left(-\frac{\alpha}{2}, 1+\gamma ;-y\right)+C_{2} t^{\gamma}\left(\frac{1}{2} \phi\left(-\frac{\alpha}{2}, 1+\gamma ; y\right)-y^{2+2 \frac{\gamma-1}{\alpha}} \Phi_{\left(2,3+2 \frac{\gamma-1}{\alpha}\right),(-\alpha, 2-\alpha)}\left(y^{2}\right)\right)
$$

in the case $1-\alpha<\gamma<1, \gamma \neq 1-\frac{\alpha}{2}, \gamma \neq 0$, and

$$
u(x, t)=C_{1} \phi\left(-\frac{\alpha}{2}, 1 ;-y\right)+C_{2}\left(\frac{1}{2} \phi\left(-\frac{\alpha}{2}, 1 ; y\right)-y^{2-\frac{2}{\alpha}} \Phi_{\left(2,3-\frac{2}{\alpha}\right),(-\alpha, 2-\alpha)}\left(y^{2}\right)\right)+C_{3}
$$

in the case $\gamma=0$, where $y=x t^{-\frac{\alpha}{2}}$ is the first scale-invariant (31), $\phi$ is the Wright function of the second kind defined by (4), $\Phi$ is the four-parameters Wright function of the second kind defined by (7), and $C_{1}, C_{2}, C_{3}$ are arbitrary constants.

For further results regarding the scale-invariant solutions to the fractional diffusion-wave equations we refer to $[9,14,15,18]$. 


\subsection{Subordination Formula for the Multi-Dimensional Space-Time-Fractional Diffusion Equations}

The object of analysis in this subsection is the multi-dimensional space-time-fractional diffusion equation

$$
D_{t}^{\beta} u(\mathrm{x}, t)=-(-\Delta)^{\frac{\alpha}{2}} u(\mathrm{x}, t), \quad \mathrm{x} \in \mathbb{R}^{n}, t>0,0<\alpha \leq 2,0<\beta \leq 1
$$

In the Equation (38), the time-fractional derivative $D_{t}^{\beta}$ is defined in the Caputo sense:

$$
D_{t}^{\beta} u(\mathrm{x}, t)=\left(I_{t}^{n-\beta} \frac{\partial^{n} u}{\partial t^{n}}\right)(t), \quad n-1<\beta \leq n, n \in \mathbb{N}
$$

with $I_{t}^{\gamma}$ being the Riemann-Liouville fractional integral:

$$
\left(I_{t}^{\gamma} u\right)(t)=\left\{\begin{array}{l}
\frac{1}{\Gamma(\gamma)} \int_{0}^{t}(t-\tau)^{\gamma-1} u(\mathrm{x}, \tau) d \tau \text { for } \gamma>0 \\
u(\mathrm{x}, t) \text { for } \gamma=0
\end{array}\right.
$$

The fractional Laplacian $-(-\Delta)^{\frac{\alpha}{2}}$ is understood as a pseudo-differential operator with the symbol $-|\kappa|^{\alpha}([38,39])$ :

$$
\left(\mathcal{F}-(-\Delta)^{\frac{\alpha}{2}} u\right)(\kappa)=-|\kappa|^{\alpha}(\mathcal{F} u)(\kappa),
$$

where $(\mathcal{F} f)(\kappa)$ is the Fourier transform of a function $u$ at the point $\kappa \in \mathbb{R}^{n}$ defined by

$$
(\mathcal{F} u)(\kappa)=\hat{f}(\kappa)=\int_{\mathbb{R}^{n}} e^{i \kappa \cdot x} u(\mathbf{x}) d \mathbf{x}
$$

The fractional Laplacian can be also represented as a hypersingular integral ([39]):

$$
-(-\Delta)^{\frac{\alpha}{2}} u(\mathrm{x})=-\frac{1}{d_{n, m}(\alpha)} \int_{\mathbb{R}^{n}} \frac{\left(\Delta_{\mathrm{h}}^{m} u\right)(\mathrm{x})}{|\mathrm{h}|^{n+\alpha}} d \mathrm{~h}, 0<\alpha<m, m \in \mathbb{N}, x \in \mathbb{R}^{n}
$$

with a suitably defined finite differences operator $\left(\Delta_{\mathrm{h}}^{m} f\right)(\mathbf{x})$ and a normalization constant $d_{n, m}(\alpha)$.

The representation (42) of the fractional Laplacian in form of the hypersingular integral does not depend on $m, m \in \mathbb{N}$ provided $\alpha<m$ ([39]). For other representations of the fractional Laplacian we refer the reader to [40].

In what follows, we consider the Cauchy problem for the space-time-fractional diffusion Equation (38) with the Dirichlet initial condition:

$$
u(\mathbf{x}, 0)=f(\mathbf{x}), \quad \mathrm{x} \in \mathbb{R}^{n}
$$

Because the initial-value problem (38), (43) is linear, its solution can be represented in the form

$$
u(\mathrm{x}, t)=\int_{\mathbb{R}^{n}} G_{\alpha, \beta, n}(\zeta, t) f(\mathrm{x}-\zeta) d \zeta
$$

In (44), the function $f$ is the initial condition and $G_{\alpha, \beta, n}$ is the first fundamental solution of (38), i.e., its solution with the initial condition

$$
u(\mathrm{x}, 0)=\prod_{i=1}^{n} \delta\left(x_{i}\right), \quad \mathrm{x}=\left(x_{1}, x_{2}, \ldots, x_{n}\right) \in \mathbb{R}^{n}
$$

where $\delta$ is the Dirac delta function. 
In the case of the conventional diffusion equation $(\alpha=2$ and $\beta=1$ in the Equation (38)), the fundamental solution is well-known:

$$
G_{2,1, n}(\mathrm{x}, t)=\frac{1}{(\sqrt{4 \pi t})^{n}} \exp \left(-\frac{|\mathrm{x}|^{2}}{4 t}\right) .
$$

It turned out that the fundamental solution $G_{\alpha, \beta, n}$ to the multi-dimensional space-time-fractional diffusion Equation (38) can be represented in terms of the fundamental solution $G_{2,1, n}$ of the conventional diffusion equation. The result obtained in [33] for the first time is given in the following theorem:

Theorem 8 ([33]). For the fundamental solution $G_{\alpha, \beta, n}(x, t)$ to the multi-dimensional space-time-fractional diffusion-wave Equation (38) with $0<\beta \leq 1,0<\alpha \leq 2$, and $2 \beta+\alpha<4$ the following subordination formula is valid:

$$
G_{\alpha, \beta, n}(\mathrm{x}, t)=\int_{0}^{\infty} t^{-\frac{2 \beta}{\alpha}} \Psi_{\alpha, \beta}\left(s t^{-\frac{2 \beta}{\alpha}}\right) G_{2,1, n}(\mathrm{x}, s) d s,
$$

where the fundamental solution $G_{2,1, n}(x, s)$ to the conventional diffusion equation is given by the formula (45) and the kernel function $\Psi_{\alpha, \beta}$ is a probability density function in $s, s \in \mathbb{R}_{+}$for each value of $t, t>0$ defined as follows:

$$
\Psi_{\alpha, \beta}(\tau)=\left\{\begin{array}{l}
\tau^{\frac{\alpha}{2}-1} \Phi_{\left(\frac{\alpha}{2}, \frac{\alpha}{2}\right),(-\beta, 1-\beta)}\left(-\tau^{\frac{\alpha}{2}}\right) \text { if } \frac{\beta}{\alpha}<\frac{1}{2}, \\
-\tau^{-1-\frac{\alpha}{2}} \Phi_{(\beta, 1-\beta),\left(-\frac{\alpha}{2},-\frac{\alpha}{2}\right)}\left(-\tau^{-\frac{\alpha}{2}}\right) \text { if } \frac{\beta}{\alpha}>\frac{1}{2}, \\
\left\{\begin{array}{l}
\frac{\tau^{\frac{\alpha}{2}-1}}{\pi} \sum_{k=0}^{\infty} \sin \left(\frac{\pi \alpha}{2}(k+1)\right)\left(-\tau^{\frac{\alpha}{2}}\right)^{k} \text { if } 0<\tau<1 \\
-\frac{\tau^{-1}}{\pi} \sum_{k=0}^{\infty} \sin \left(\frac{\pi \alpha}{2} k\right)\left(-\tau^{-\frac{\alpha}{2}}\right)^{k} \text { if } \tau>1
\end{array} \text { if } \frac{\beta}{\alpha}=\frac{1}{2} .\right.
\end{array}\right.
$$

In the formula (47), the function $\Phi$ is the four-parameters Wright function of the second kind defined by (9).

It is worth mentioning that even if the subordination formula (46) concerns just the fundamental solution, it can be extended to the solution operator for the initial-value problem (38), (43). Indeed, let us suppose that a more general subordination formula for the fundamental solution $G_{\alpha, \beta, n}$ is valid:

$$
G_{\alpha, \beta, n}(\mathrm{x}, t)=\int_{0}^{\infty} \Psi(\alpha, \beta, s, t) G_{\hat{\alpha}, \hat{\beta}, n}(\mathrm{x}, s) d s,
$$

where the kernel function $\Psi=\Psi(\alpha, \beta, s, t)$ can be interpreted as a probability density function in $s, s \in \mathbb{R}_{+}$for each value of $t, t>0$ (the formula (47) is a particular case of the formula (48)). Then we have the following chain of relations:

$$
\begin{gathered}
S_{\alpha, \beta, n}(t) f=\int_{\mathbb{R}^{n}} G_{\alpha, \beta, n}(\zeta, t) f(\mathrm{x}-\zeta) d \zeta=\int_{\mathbb{R}^{n}} \int_{0}^{\infty} \Psi(\alpha, \beta, s, t) G_{\hat{\alpha}, \hat{\beta}, n}(\zeta, s) d s f(\mathrm{x}-\zeta) d \zeta= \\
\int_{0}^{\infty} \Psi(\alpha, \beta, s, t) \int_{\mathbb{R}^{n}} G_{\hat{\alpha}, \hat{\beta}, n}(\zeta, s) f(\mathrm{x}-\zeta) d \zeta d s=\int_{0}^{\infty} \Psi(\alpha, \beta, s, t) S_{\hat{\alpha}, \hat{\beta}, n}(s) f d s .
\end{gathered}
$$

Thus, the subordination formula

$$
S_{\alpha, \beta, n}(t) f=\int_{0}^{\infty} \Psi(\alpha, \beta, s, t) S_{\hat{\alpha}, \hat{\beta}, n}(s) f d s
$$


holds true for the solution operator $S_{\alpha, \beta, n}$. Vice versa, any subordination formula for the solution operator $S_{\alpha, \beta, n}$ to the initial-value problem (38), (43) in the form (49) induces a subordination formula of the type (48) for the fundamental solution $G_{\alpha, \beta, n}$ just by setting $f$ to be the Dirac $\delta$-function.

In the rest of this subsection, we provide some important remarks concerning the kernel $\Psi_{\alpha, \beta}$ of the subordination formula (46).

In [33], the kernel function $\Psi_{\alpha, \beta}$ given by the formula (47) was first deduced in form of the following Mellin-Barnes integral:

$$
\Psi_{\alpha, \beta}(\tau)=\frac{2}{\alpha} \frac{1}{2 \pi i} \int_{\gamma-i \infty}^{\gamma+i \infty} \frac{\Gamma\left(\frac{2}{\alpha}-\frac{2}{\alpha} s\right) \Gamma\left(1-\frac{2}{\alpha}+\frac{2}{\alpha} s\right)}{\Gamma\left(1-\frac{2 \beta}{\alpha}+\frac{2 \beta}{\alpha} s\right) \Gamma(1-s)} \tau^{-s} d s .
$$

The series representation (47) was derived by evaluating the Mellin-Barnes integral (50) taking into account the Jordan lemma, the formula (14) for the residual of the Gamma-function $\Gamma(s)$ at the point $s=-k$, the asymptotic behavior of the Gamma-function, and the Cauchy residue theorem.

The kernel function $\Psi_{\alpha, \beta}$ can be also interpreted as the inverse Laplace transform of the Mittag-Leffler function $E_{\beta}\left(-\lambda^{\frac{\alpha}{2}}\right)$ :

$$
E_{\beta}\left(-\lambda^{\frac{\alpha}{2}}\right)=\int_{0}^{\infty} \Psi_{\alpha, \beta}(\tau) e^{-\lambda \tau} d \tau,
$$

where the Mittag-Leffler function $E_{\beta}$ is defined as follows:

$$
E_{\beta}(z)=E_{\beta, 1}(z)=\sum_{k=0}^{\infty} \frac{z^{k}}{\Gamma(1+\beta k)}, \beta>0, z \in \mathbb{C}
$$

For the time-fractional diffusion equation $(\alpha=2,0<\beta \leq 1$ in the Equation (38)) the subordination formula (46) with the kernel function $\Phi_{\alpha, \beta}$ given by the 1st line of (47) is valid. In this case, the four-parameters Wright function of the second kind is reduced to the Wright function of the second kind and we arrive at the known formula $([41,42])$

$$
G_{2, \beta, n}(\mathrm{x}, t)=\int_{0}^{\infty} t^{-\beta} \phi\left(-\beta, 1-\beta ;-s t^{-\beta}\right) G_{2,1, n}(\mathrm{x}, s) d s, 0<\beta<1 .
$$

For the space-fractional diffusion equation $(\beta=1,0<\alpha \leq 2$ in the Equation (38)), the subordination formula (46) with the kernel function $\Psi_{\alpha, \beta}$ given by the 2 nd line of (47) is valid. It is easy to verify that the kernel function can be rewritten in the following form:

$$
-\tau^{-1-\frac{\alpha}{2}} \Phi_{(\beta, 1-\beta),\left(-\frac{\alpha}{2},-\frac{\alpha}{2}\right)}\left(-\tau^{-\frac{\alpha}{2}}\right)=\tau^{-1} \Phi_{(\beta, 1),\left(-\frac{\alpha}{2}, 0\right)}\left(-\tau^{-\frac{\alpha}{2}}\right) .
$$

Thus, also in the case of the space-fractional diffusion equation, the four-parameters Wright function of the second kind from the formula (47) is reduced to the Wright function of the second kind and we arrive at the subordination formula in the form

$$
G_{\alpha, 1, n}(\mathrm{x}, t)=\int_{0}^{\infty} s^{-1} \phi\left(-\frac{\alpha}{2}, 0 ;-s^{-\frac{\alpha}{2}} t\right) G_{2,1, n}(\mathrm{x}, s) d s, 0<\alpha<2 .
$$

\subsection{FDEs with the Left-and Right-Hand Sided Erdélyi-Kober Fractional Derivatives}

In this part of the section, we consider an initial-value problem for an ordinary fractional differential equation with the left- and right-hand sided Erdélyi-Kober fractional derivatives defined on the positive semi-axis. The equations of this type appear in the fractional calculus of variations as the Euler-Lagrange equations. However, to the best knowledge of the author, the only method for analytical treatment of these equations defined on an infinite interval, say, on the positive real semi-axis, 
is the operational method recently suggested in [32]. Here we present an example of application of this method to the following sample equation $(a>b>0, n-1<a \mu \leq n, n \in \mathbb{N})$ :

$$
\left({ }_{*} D_{1 / a}^{-\alpha-a \mu, a \mu} y\right)(x)+\rho x^{\mu}\left({ }_{*} P_{1 / b}^{\beta-b \mu, b \mu} y\right)(x)=f(x), x>0, \rho>0
$$

subject to the initial conditions $(k=0, \ldots, n-1)$

$$
\lim _{x \rightarrow 0} x^{\frac{1}{a}(1-\alpha-a \mu+k)} \prod_{i=k+1}^{n-1}\left(1-\alpha-a \mu+i+a x \frac{d}{d x}\right) y(x)=c_{k} .
$$

In the Equation (55), the operator ${ }_{*} P_{1 / b}^{\beta-b \mu, b \mu}$ is the Caputo type modification of the right-hand sided Erdélyi-Kober fractional derivative given by the formula (34). The operator ${ }_{*} D_{1 / a}^{-\alpha-a \mu, a \mu}$ is the Caputo type modification of the left-hand sided Erdélyi-Kober fractional derivative defined as follows:

$$
\left({ }_{*} D_{\beta}^{\gamma, \delta} f\right)(x)=\left(I_{\beta}^{\gamma+\delta, n-\delta} \prod_{k=0}^{n-1}\left(1+\gamma+k+\frac{1}{\beta} t \frac{d}{d t}\right) f\right)(x),
$$

where $I_{\beta}^{\gamma, \delta}$ stays for the left-hand sided Erdélyi-Kober fractional integral of order $\delta$ :

$$
\left(I_{\beta}^{\gamma, \delta} f\right)(x)=\frac{1}{\Gamma(\delta)} \int_{0}^{1}(1-t)^{\delta-1} t^{\gamma} f\left(x t^{\frac{1}{\beta}}\right) d t, \delta, \beta>0, \gamma \in \mathbb{R}
$$

It is worth mentioning that the initial conditions in form (56) are determined by the projector operator of the left-hand sided Erdélyi-Kober fractional integral $I_{1 / a}^{-\alpha-a \mu, a \mu}$

$$
\begin{gathered}
(P y)(x)=y(x)-\left(I_{1 / a}^{-\alpha-a \mu, a \mu}{ }_{*} D_{1 / a}^{-\alpha-a \mu, a \mu} y\right)(x)=\sum_{k=0}^{n-1} c_{k} x^{-\frac{1}{a}(1-\alpha-a \mu+k),} \\
c_{k}=\lim _{x \rightarrow 0} x^{\frac{1}{a}(1-\alpha-a \mu+k)} \prod_{i=k+1}^{n-1}\left(1-\alpha-a \mu+i+a x \frac{d}{d x}\right) y(x)
\end{gathered}
$$

and thus, they are quite natural for the Equation (38).

In this paper, we do not repeat the derivation of the exact solution to the initial-value problem (55), (56) presented in [32] and restrict ourselves to formulation of the final result.

Theorem 9. Let $a>b>0, n-1<a \mu \leq n, n \in \mathbb{N}, f \in \mathfrak{O}$, and the condition

$$
\frac{\alpha-1}{a}<\frac{\beta}{b}
$$

be satisfied. Then the initial-value problem (38), (56) possesses a unique solution on the space $\mathfrak{O}$ in the form

$$
y(x)=\sum_{k=0}^{n-1} c_{k} y_{k}(x)+y_{f}(x)
$$

where the functions $y_{k}, k=0, \ldots, n-1$ are defined by

$$
y_{k}(x)=\Gamma(a \mu-k) \Gamma\left(\beta+\frac{b}{a}(1-\alpha-a \mu+k)\right) x^{\mu-\frac{1}{a}(1-\alpha+k)} \Phi_{(a \mu, a \mu-k),\left(-b \mu, \beta+\frac{b}{a}(1-\alpha-a \mu+k)\right.}\left(-\rho x^{\mu}\right),
$$

and the function $y_{f}$ is given by the formula

$$
y_{f}(x)=g(x)+\left(g^{*} * y_{\Phi}\right)(x)
$$


with

$$
g(x)=\left(I_{1 / a}^{-\alpha-a \mu, a \mu} f\right)(x), y_{\Phi}(x)=\rho x^{\mu-\lambda} \Phi_{(a \mu, 1-\alpha+a(\mu-\lambda)),(-b \mu, \beta-b(\mu-\lambda)}\left(-\rho x^{\mu}\right)
$$

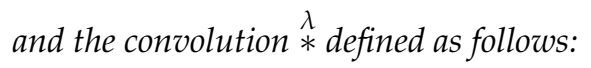

$$
(f * g)(x)=\left(I_{1 / a}^{1-2 \alpha-a \lambda, \alpha+a \lambda-1} * P_{1 / b}^{\beta, \beta+b \lambda} f \circ g\right)(x)
$$

with

$$
(f \circ g)(x)=x^{\lambda} \int_{0}^{1} \int_{0}^{1} \tau_{1}^{-\alpha}\left(1-\tau_{1}\right)^{-\alpha} \tau_{2}^{\beta-1}\left(1-\tau_{2}\right)^{\beta-1} f\left(\frac{x \tau_{1}^{a}}{\tau_{2}^{b}}\right) g\left(\frac{x\left(1-\tau_{1}\right)^{a}}{\left(1-\tau_{2}\right)^{b}}\right) d \tau_{1} d \tau_{2} .
$$

The function $y_{f}$ satisfies the inhomogeneous Equation (55) and homogeneous initial conditions, whereas the functions $y_{k}, k=0, \ldots, n-1$ satisfy the homogeneous Equation (55) $(f(x) \equiv 0, x>0)$ and the initial conditions $(k=0, \ldots, n-1, j=0, \ldots, n-1)$

$$
\lim _{x \rightarrow 0} x^{\frac{1}{a}(1-\alpha-a \mu+j)} \prod_{i=j+1}^{n-1}\left(1-\alpha-a \mu+i+a x \frac{d}{d x}\right) y_{k}(x)=\left\{\begin{array}{l}
1, j=k, \\
0, j \neq k .
\end{array}\right.
$$

In the formulation of the theorem, the space of functions denoted by $\mathfrak{O}$ consists of the functions that are continuous on the semi-axis ] $0, \infty[$ and can be represented as the convergent power series with the power functions weights in some neighborhoods $U_{\epsilon_{1}}(0)$ and $U_{\epsilon_{2}}(+\infty)$ of the points $x=0$ and $x=+\infty$, respectively, i.e., in the form

$$
f(x)=x^{\alpha} \sum_{k=0}^{\infty} a_{k}\left(x^{\rho}\right)^{k}, \rho>0, x \in U_{\epsilon_{1}}(0),
$$

and

$$
f(x)=x^{\beta} \sum_{k=0}^{\infty} b_{k}\left(x^{-\sigma}\right)^{k}, \sigma>0, x \in U_{\epsilon_{2}}(+\infty) .
$$

The functions from $\mathfrak{O}$ have a power law asymptotic behavior at the points 0 and $+\infty$ that appears to be an appropriate asymptotics for solutions of the fractional differential equations that contain both the left- and right-hand sided Erdélyi-Kober fractional derivatives.

Finally, we mention that the results formulated in Theorem 9 remain valid also in the case of the Equation (55) with a negative parameter $\rho$ under the additional condition $a / 3<b$. This can be proved by the operational method presented in [32] and employing the asymptotic behavior of the four-parameters Wright function of the second kind on the positive semi-axis given in Theorem 4 .

Funding: This research received no external funding.

Conflicts of Interest: The author declares no conflict of interest.

\section{References}

1. Prudnikov, A.P.; Brychkov, Y.A.; Marichev, O.I. Integrals and Series. More Special Functions; Gordon and Breach: New York, NY, USA, 1989; Volume 3.

2. Meijer, C.S. On the G-function. I, II, III, IV, V, VI, VII, VIII. Proc. Nederl. Akad. Wet. 1946, 49, 227-237, 344-356, 457-469, 632-641, 765-772, 936-943, 1063-1072, 1165-1175.

3. Gorenflo, R.; Kilbas, A.A.; Mainardi, F.; Rogosin, S.V. Mittag-Leffler Functions, Related Topics and Applications; Springer: Berlin, Germany, 2014.

4. Kiryakova, V. Generalized Fractional Calculus and Applications; J. Wiley: New York, NY, USA, 1994. 
5. Luchko, Y. The Wright function and its applications. In Handbook of Fractional Calculus with Applications. Vol.1: Basic Theory; Kochubei, A., Luchko, Y., Eds.; Walter de Gruyter: Berlin, Germany; Boston, MA, USA, 2019; pp. 241-268.

6. Paris, R.B. Asymptotics of the special functions of fractional calculus. In Handbook of Fractional Calculus with Applications. Vol. 1: Basic Theory; Kochubei, A., Luchko, Y., Eds.; Walter de Gruyter: Berlin, Germany; Boston, MA, USA, 2019; pp. 297-326.

7. Srivastava, H.M.; Gupta, K.C.; Goyal, S.P. The H-functions of One and Two Variables with Applications; South Asian Publishers: New Delhi, India, 1982.

8. Gorenflo, R.; Mainardi, F.; Rogosin, S. Mittag-Leffler function: Properties and applications. In Handbook of Fractional Calculus with Applications. Vol.1: Basic Theory; Kochubei, A., Luchko, Y., Eds.; Walter de Gruyter: Berlin, Germany; Boston, MA, USA, 2019; pp. 269-298.

9. Buckwar, E.; Luchko, Y. Invariance of a partial differential equation of fractional order under the Lie group of scaling transformations. J. Math. Anal. Appl. 1998, 227, 81-97. [CrossRef]

10. Consiglio, A.; Mainardi, F. The Wright function of the second kind in mathematical physics. Mathematics 2020, 8,884 .

11. Das, S.; Mehrez, K. Geometric properties of the four parameters Wright function. arXiv 2020, arXiv:2005.01354v1.

12. Djrbashian, M.M. On integral transforms generated by the generalized Mittag-Leffler function. Izv. Akad. Nauk Armjan. SSR. 1960, 13, 21-63. (In Russian)

13. Gajic̀, L.; Stankovic̀, B. Some properties of Wright's function. Publ. de l'Institut Mathèmatique, Beograd, Nouvelle Sèr. 1976, 20, 91-98.

14. Gorenflo, R.; Luchko, Y.; Mainardi, F. Analytical properties and applications of the Wright function. Fract. Calc. Appl. Anal. 1999, 2, 383-414.

15. Gorenflo, R.; Luchko, Y.; Mainardi, F. Wright functions as scale-invariant solutions of the diffusion-wave equation. J. Computat. Appl. Math. 2000, 11, 175-191. [CrossRef]

16. Kilbas, A.A.; Saigo, M.; Trujillo, J.J. On the generalized Wright function. Fract. Calc. Appl. Anal. 2002, 5, 437-460.

17. Luchko, Y. On the asymptotics of zeros of the Wright function. Zeitschrift für Analysis und ihre Anwendungen 2000, 19, 597-622. [CrossRef]

18. Luchko, Y.; Gorenflo, R. Scale-invariant solutions of a partial differential equation of fractional order. Fract. Calc. Appl. Anal. 1998, 1, 63-78.

19. Mainardi, F. Fractional Calculus and Waves in Linear Viscoelasticity; Imperial College Press: London, UK, 2010.

20. Mainardi, F.; Pagnini, G. The Wright functions as solutions of the time-fractional diffusion equations. Appl. Math. Comp. 2003, 141, 51-62. [CrossRef]

21. Mainardi, F.; Pagnini, G. The role of the Fox-Wright functions in fractional sub-diffusion of distributed order. J. Comput. App. Math. 2007, 207, 245-257. [CrossRef]

22. Mehrez, K. New Integral representations for the Fox-Wright functions and its applications. arXiv 2017, arXiv:1711.08368.

23. Mehrez, K. Monotonicity patterns and functional inequalities for classical and generalized Wright functions. arXiv 2017, arXiv:1708.00461.

24. Mehrez, K.; Sitnik, S.M. Functional inequalities for Fox-Wright functions. arXiv 2017, arXiv:1708.06611.

25. Stankovic̀, B. On the function of E.M. Wright. Publications De l'Institut Mathèmatique 1970, 10, 113-124.

26. Wright, E.M. On the coefficients of power series having exponential singularities. J. Lond. Math. Soc. 1933, 8 , 71-79. [CrossRef]

27. Wright, E.M. The asymptotic expansion of the generalized Bessel function, Proc. London Math. Soc. (Ser. II) 1935, 38, 257-270. [CrossRef]

28. Wright, E.M. The asymptotic expansion of the generalized hypergeometric function. J. Lond. Math. Soc. 1935, 10, 287-293. [CrossRef]

29. Wright, E.M. The generalized Bessel function of order greater than one. Quart. J. Math. Oxford Ser. 1940, 11, 36-48. [CrossRef]

30. Fox, C. The asymptotic expansion of generalized hypergeometric functions. Proc. Lond. Math. Soc. 1928, 27, 389-400. [CrossRef]

31. Gorenflo, R.; Luchko, Y.; Srivastava, H.M. Operational method for solving integral equations with Gauss's hypergeometric function as a kernel. Int. J. Math. Stat. Sci. 1997, 6, 179-200. 
32. Hanna, L.A.-M.; Al-Kandari, M.; Luchko, Y. Operational method for solving fractional differential equations with the left-and right-hand sided Erdélyi-Kober fractional derivatives. Fract. Calc. Appl. Anal. 2020, 23, 103-125. [CrossRef]

33. Luchko, Y. Subordination principles for the multi-dimensional space-time-fractional diffusion- wave equation. Theor. Probab. Math. Statist. 2019, 98, 127-147. [CrossRef]

34. Kilbas, A.A.; Srivastava, H.M.; Trujillo, J.J. Theory and Applications of Fractional Differential Equations; Elsevier: Amsterdam, The Netherlands, 2006.

35. Marichev, O.I. Handbook of Integral Transforms of Higher Transcendental Functions. Theory and Algorithmic Tables; Ellis Horwood: Chichester, UK, 1983.

36. Mainardi, F.; The fundamental solutions for the fractional diffusion-wave equation. Appl. Math. Lett. 1996, 9 , 23-28. [CrossRef]

37. Olver, P.J. Applications of Lie Groups to Differential Equations; Springer: New York, NY, USA, 1986.

38. Saichev, A.; Zaslavsky, G. Fractional kinetic equations: Solutions and applications. Chaos 1997, 7, 753-764. [CrossRef]

39. Samko, S.G.; Kilbas, A.A.; Marichev, O.I. Fractional Integrals and Derivatives: Theory and Applications; Gordon and Breach: New York, NY, USA, 1993.

40. Kwaśnicki, M. Fractional Laplace operator and its properties. In Handbook of Fractional Calculus with Applications. Vol. 1: Basic Theory; Kochubei, A., Luchko, Y., Eds.; Walter de Gruyter: Berlin, Germany; Boston, MA, USA, 2019; pp. 159-194.

41. Bazhlekova, E. Subordination principle for fractional evolution equations. Fract. Calc. Appl. Anal. 2000, 3, 213-230.

42. Bajlekova, E. Fractional Evolution Equations in Banach Spaces. Ph.D. Thesis, Technische Universiteit Eindhoven, Eindhoven, The Netherlands, 2001.

(C) 2020 by the authors. Licensee MDPI, Basel, Switzerland. This article is an open access article distributed under the terms and conditions of the Creative Commons Attribution (CC BY) license (http://creativecommons.org/licenses/by/4.0/). 